\title{
Factores relacionados a candidiasis oral en niños y adolescentes con VIH, caracterización de especies y susceptibilidad antifúngica
}

\author{
Nydia A. Castillo-Martínez', Rosa R. Mouriño-Pérez², José M. Cornejo-Bravo3 y Luis A. Gaitán-Cepeda
}

\section{Factors related to oral candidiasis in HIV children and adolescents, species characterization and antifungal susceptibility}

Background: Factors associated with candidiasis and colonization in HIV-positive children and adolescents in developing countries are not well understood. Aim: To identify the factors associated with oral Candida colonization and candidiasis in institutionalized HIV-positive children and adolescents in Tijuana, México, as well as the response of the isolates to antifungals. Materials and Methods: Sample of the oral mucosa of 30 HIV positive children and adolescents were obtained to isolate and identify Candida species by culture and metabolic profile. Antifungal drugs susceptibility was determined according to CLSI. Indicators of immunological and virologic failure were classified in accordance to WHO criteria. Results: Six Candida species were identified from oral mucosa, $53 \%$ colonizers and $47 \%$ in candidiasis. Factors associated with candidiasis and oral colonization were viral load $(p=0,001)$, CD4+ counts $(p=0,002)$ and HAART regimen $(p \leq 0,014)$. The most prevalent species was $C$. glabrata $(33 \%)$, but $C$. albicans $(27 \%)$ was more resistant to fluconazole $(\mathrm{p}=0,001)$. Itraconazol resistant species were identified in regimens that include an NNRTI $(\mathrm{p}=0,041)$. Conclusion: HIV-positive children and adolescents living in an orphanage showed high prevalence of colonizing Candida spp. and resistance to antifungals, related to NNRTI.

Key words: Highly active antiretroviral therapy; oral candidiasis; children; HIV.

Palabras clave: Tratamiento anti-retroviral de alta actividad; candidiasis oral; niños; VIH.

\section{Introducción}

$\mathrm{E}$ n América Latina y el Caribe hay aproximadamente de 2 millones de personas infectadas con el virus de la inmunodeficiencia humana (VIH), de las cuales 2.100 son casos nuevos pediátricos ${ }^{1}$. En México, hasta el año 2016, se contabilizaron 64.106 personas con infección por VIH, de las cuales 4.571 tenían menos de 19 años de edad (7,13\%). La principal vía de transmisión en la población de 15 a 19 años es la sexual (95\%) y menos de 15 años de edad es la perinatal $(88 \%)^{2}$. En 2016 en el Estado de Baja California, México, había 2.410 personas con infección por VIH, lo que corresponde a $3,8 \%$ de los casos de todo el país ${ }^{3}$.

La candidiasis orofaríngea es la principal infección oral oportunista asociada a la infección por VIH en niños $\mathrm{y}$ adolescentes, directamente asociada a recuentos bajos de linfocitos T CD4+ (LTCD4+) en sangre periférica, por debajo de 200 céls/ $\mu \mathrm{L}$ por lo que su presencia se ha utilizado como un marcador clínico de la infección $n^{4,5}$.

Las infecciones oportunistas han disminuido en los países desarrollados desde la introducción del tratamiento anti-retroviral de alta actividad (TARAA); sin embargo, en los países en vías de desarrollo no han mostrado el mismo impacto y la información acerca de los factores involucrados en la presencia de las manifestaciones clínicas es inconclusa ${ }^{6}$. La utilidad de las infecciones orales oportunistas como predictores de falla terapéutica en población pediátrica infectada por VIH no ha sido establecida, de manera particular en países de Latinoamérica donde aún se tiene alta prevalencia de transmisión madre a hijo y, por lo tanto, alta incidencia de casos en infantes ${ }^{7-11}$.

Por otra parte, no ha sido totalmente esclarecido el efecto del TARAA sobre la susceptibilidad a antifúngicos de las especies de Candida. La interacción entre ambos conduce a una resistencia clínica hacia los antifúngicos $y$, por otra parte, la TARAA puede activar factores de virulencia de los microorganismos colonizantes ocasionando una resistencia a los antifúngicos por mecanismos celulares o moleculares ${ }^{12}$. Un ejemplo de este último efecto es la actividad de los inhibidores de proteasa (IP) del VIH sobre las aspartil-proteasas que Candida utiliza como factor de virulencia para adherirse a la mucosa del hospedero ${ }^{13,14}$.

Este estudio se realizó con el objetivo de conocer la prevalencia de especies de Candida en la cavidad oral de
'Laboratorio de Microbiología. Escuela de Ciencias de la Salud, Valle de las Palmas. Universidad Autónoma de Baja California, México.

2Departamento de Microbiología. Centro de Investigación Científica y de Educación Superior de Ensenada. Ensenada B.C. ${ }^{3}$ Facultad de Ciencias Químicas e Ingeniería. Universidad Autónoma de Baja California.

${ }^{4}$ Laboratorio de Patología Clínica y Experimental de la División de Estudios de Posgrado e Investigación. Facultad de Odontología UNAM.

Fuente de financiamiento: 17 Convocatoria Interna de Proyectos de Investigación de la Universidad Autónoma de Baja California, México.

Los autores declaran no tener conflicto de interés.

Recibido: 18 de agosto de 2017 Aceptado: 26 de junio de 2018

Correspondencia a: Nydia A. Castillo-Martínez nydia.castillo@uabc.edu.mx 
niños y adolescentes infectados por VIH, institucionalizados de la ciudad de Tijuana y los patrones de sensibilidad a los antifúngicos de las cepas aisladas. Adicionalmente se establecerán posibles asociaciones entre las características demográficas, marcadores de laboratorio, estado inmunológico y tratamiento anti-retroviral de los individuos, la colonización por especies de Candida y la candidiasis oral.

\section{Material y Métodos}

Se realizó un estudio transversal, prospectivo, en 30 niños y adolescentes con infección por VIH, entre 2 y 19 años de edad, durante el año 2014. Todos los pacientes al momento de su revisión estaban institucionalizados en un orfanato para menores con infección por VIH, cuya autoridad otorgó la aprobación y el consentimiento informado para el estudio.

La atención médica y el seguimiento estaban a cargo del Servicio de Pediatría e Infectología del Hospital General de Tijuana.

Durante la revisión bucal, se registraron los datos demográficos y de antropometría. Además, se solicitó la información de la terapia anti-retroviral e historia clínica de otras manifestaciones debidas al VIH, así como de los tratamientos previos por oportunistas.

La carga viral (CV) y cuantificación de LTCD4+ se realizaron en un laboratorio certificado de la ciudad.

Para el aislamiento de especies de Candida se recolectaron muestras de la mucosa oral total con un hisopo estéril en un medio de transporte de Stuart y se trasladaron al Laboratorio de Microbiología de la Escuela de Ciencias de la Salud-Valle de las Palmas, de la Universidad Autónoma de Baja California.

Para la identificación, se inocularon en placas de agar cromogénico para Candida (HardyChrom ${ }^{\mathrm{TM}}$ Candida, HardyDiagnostics) y Sabouraud dextrosa (BBL ${ }^{\mathrm{TM}}, \mathrm{BD}$ Diagnostics). Se incubaron a $37{ }^{\circ} \mathrm{C}$ durante $48 \mathrm{~h}$ y 25 ${ }^{\circ} \mathrm{C}$ por $24 \mathrm{~h}$. Se obtuvieron cultivos axénicos en placas de agar Sabouraud dextrosa con cloranfenicol (CRITERIONTM, HardyDiagnostics) incubadas a $37^{\circ} \mathrm{C}$ durante $48 \mathrm{~h}$ y $25^{\circ} \mathrm{C}$ durante $24 \mathrm{~h}$. La especie se identificó por auxonograma utilizando el panel para la identificación rápida de levaduras (Rapid Yeast ID, Beckman Coulter, Inc). La concentración inhibitoria mínima (CIM) de los antifúngicos flucitosina (5FC), anfotericina $\mathrm{B}$ (AMB), fluconazol (FCA), itraconazol (ITR) y voriconazol (VOR) se determinaron con la galería de pruebas ATB Fungus $3^{\mathrm{TM}}$ (BioMérieux, Francia), siguiendo las recomendaciones del fabricante. Para el control de calidad se utilizaron cepas de Candida albicans ATCC $^{\circledR} 90028$, Candida parapsilosis ATCC ${ }^{\circledR} 22019$ y Candida krusei (Issatchenkia orientalis) $\mathrm{ATCC}^{\circledR}$ 6258. Las lecturas visuales y los patrones de susceptibilidad se confirmaron por el método de referencia del Clinical and Laboratory Standards Institute (CLSI) para microdilución en caldo ${ }^{15}$. La interpretación de los puntos de corte clínicos de la CIM para FCA y VOR se estableció de acuerdo a los estándares del CLSI para las pruebas de sensibilidad a los antifúngicos en levaduras ${ }^{16}$, así como los puntos de corte epidemiológicos (epidemiological cut-off value-ECV por sus siglas en inglés) para 5FC, AMB, ITR y VOR para $C$. glabrata $^{17}$. Los estándares del CLSI no indican puntos de corte para C. lipolytica y C. kefyr.

\section{Análisis estadístico}

Las variables estudiadas fueron género, edad, grupos de edad, CV y concentración de LTCD4+; estadio cínico, las manifestaciones orales, candidiasis oral, caries, estomatitis aftosa y absceso recurrente; el esquema TARAA y el tiempo de terapia ARV ${ }^{18,19}$.

Los casos de infección por VIH/SIDA de niños y adolescentes se clasificaron en tres estadios clínicos relacionados a la sintomatología de la infección por VIH y el recuento de LTCD4+. En el estadio 1 se agruparon los niños menores de 6 años con recuento de LTCD4+ $\geq 1.000$ céls $/ \mu \mathrm{L}$, y mayores de 6 años con recuento de LTCD4 $+\geq 500$ céls $/ \mu \mathrm{L}$. En el estadio 2 se agruparon a los pacientes bajo 6 años de edad con recuento de LTCD4+ entre 500 y 999 céls $/ \mu \mathrm{L}$, y mayores de 6 años con recuento de LTCD4+ entre 200 y 499 céls/ $\mu$ L. En el estadio 3 se agrupó a los niños bajo 6 años con LTCD4+ $\leq 500$ céls/ $\mu \mathrm{L}$ y niños sobre 6 años con recuento de LTCD4+ $\leq 200$ céls/ $\mu \mathrm{L}$, quienes además presentaban enfermedades oportunistas relacionadas a la infección por $\mathrm{VIH}^{19,20}$. Los criterios de clasificación para los adolescentes se establecieron de acuerdo las infecciones por microrganismos oportunistas asociadas a la infección por $\mathrm{VIH}^{19}$. La colonización por especies de Candida y la candidiasis oral se agruparon en relación a los resultados de los cultivos y las manifestaciones orales.

Colonización se definió como la presencia de especies de Candida en placa de agar (cultivo positivo) sin la manifestación clínica de candidiasis oral. Candidiasis oral de definió como el cultivo positivo acompañado de la manifestación clínica.

Los niños y adolescentes sin recibir TARAA en algún momento de su vida se consideraron naïve.

Para el análisis de los datos se utilizó el paquete estadístico STATA ver. 13 (StataCorp LLC). En el estudio de asociación entre variables categóricas se aplicó la prueba exacta de Fisher con un nivel de significancia de $0,05(\mathrm{p}<0,05)$ a dos colas. Se realizó regresión múltiple para observar la posibilidad de que ocurran las manifestaciones orales debido a la influencia entre los factores de exposición. Se calculó la razón de productos cruzados (odds ratio; OR) mediante análisis de regresión logística 
exacta, con un nivel de significancia de $0,05(\mathrm{p}<0,05)$ e intervalo de confianza de $95 \%$, a una y dos colas utilizando las frecuencias de los casos.

\section{Resultados}

Se analizaron 30 muestras provenientes de la mucosa oral de 20 niños y 10 adolescentes. Se pudo aislar Candida spp. (Tabla 1) en 15 de ellos: 6 hombres (20\%) y 9 mujeres (30\%). Promedio de edad: 8,8 años (DE $\pm 6,2$ ) y una prevalencia de $50 \%$. Se identificaron seis especies de Candida: C. glabrata (33\%), C. albicans (27\%), C. kefyr (13\%), C. krusei (13\%), C. lipolytica (7\%) y $C$. tropicalis $(7 \%)$.

\section{Hallazgo de Candida y características clínicas y de laboratorio en la población estudiada}

De los 15 aislados de Candida, 8 casos (53\%) correspondieron a portadores asintomáticos (Candida sp. colonizante) y $7(47 \%)$ a sujetos con candidiasis oral (Tabla 2). No se encontraron diferencias entre especies de Candida colonizantes o provenientes de sujetos con candidiasis oral $(p \geq 0,05)$. La forma clínica más frecuente de candidiasis fue la pseudomembranosa con $6(20 \%)$ aislamientos $(p=0,009)$.

Se observaron diferencias entre los resultados del cultivo y la carga viral $(\mathrm{p}=0,04)$. Cincuenta por ciento de las especies de Candida se aislaron de sujetos con recuentos de LTCD4+ $\geq 1.065$ céls $/ \mu \mathrm{L}(\mathrm{p}=0,02)$. Los

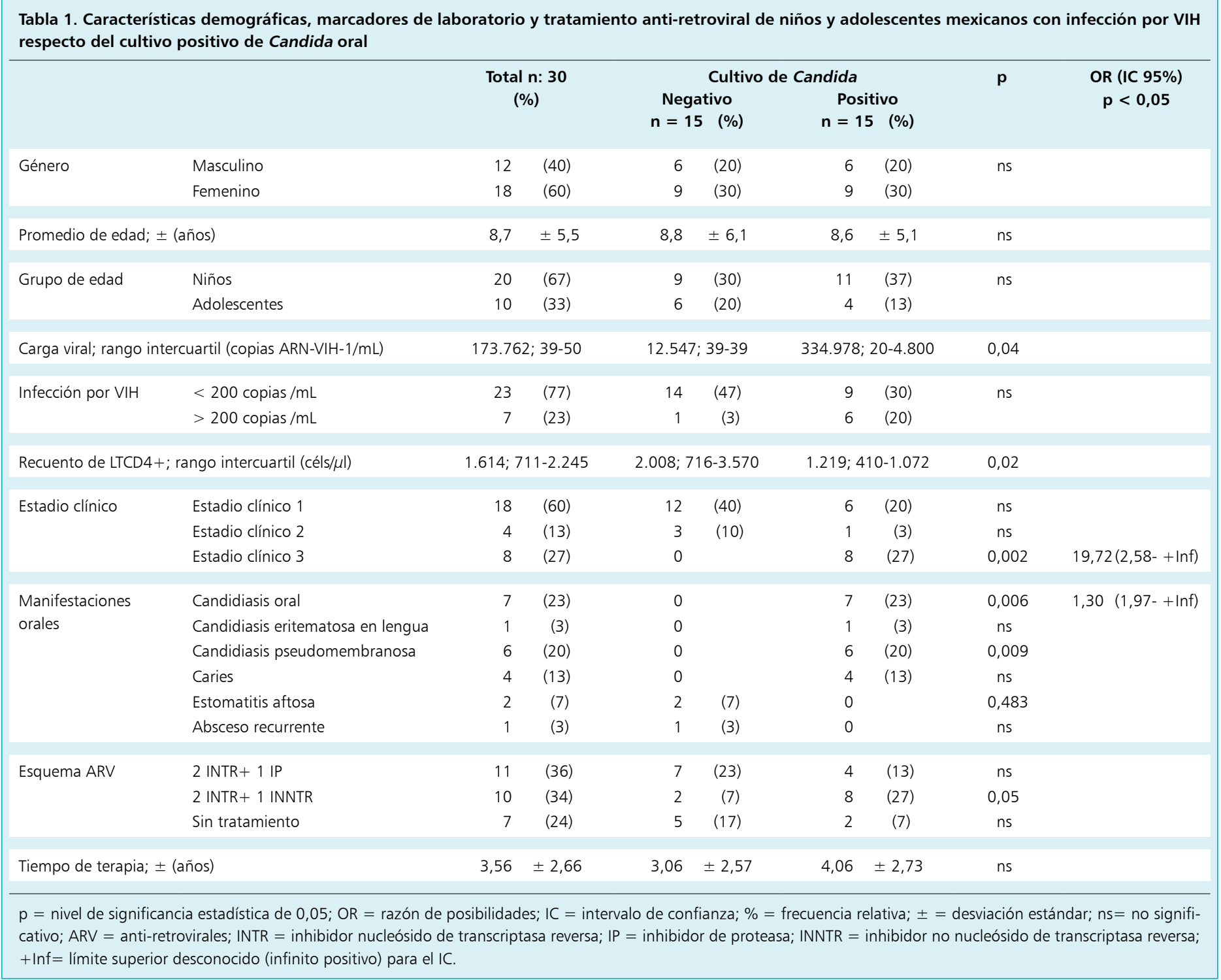


aislados provenientes de pacientes con candidiasis oral se observaron en sujetos con recuentos bajos de LTCD4+ $(610,14 \pm 500,22)$, mientras que las especies colonizantes se observaron principalmente en sujetos con recuentos de LTCD4+ sobre la mediana $(\mathrm{p}=0,002)$.

De igual manera, hubo diferencias en la $\mathrm{CV}$ de niños y adolescentes que eran portadores asintomáticos y los que tenían infección oral por Candida $(\mathrm{p}=0,004)$.

En relación al estadio clínico, 6 casos $(20 \%)$ del estadio clínico 1 y en todos los casos del estadio clínico $3(\mathrm{p}=0,002)$ tuvieron cultivo positivo. Con respecto a la terapia antirretroviral $23(77 \%)$ de los sujetos incluidos estaban bajo esquema TARAA, y 7 no reportaron estar bajo tratamiento anti-retroviral alguno. No se encontró asociación entre esquemas TARAA y aislamiento de Candida $(\mathrm{p} \geq 0,05)$.

El 33\% de esta población pediátrica se encontraba bajo esquema que incluía un inhibidor no nucleósido de la transcriptasa reversa (INNTR). Los esquemas antiretrovirales mostraron independencia en su relación con la candidiasis oral y la colonización $(\mathrm{p}=0,014)$, en particular con el esquema que incluye al INNTR, presente en $40 \%$ de casos de candidiasis oral $(\mathrm{p}=0,041)$.

Los factores asociados a los resultados del cultivo fueron (Tabla 1): la candidiasis oral $(\mathrm{p}=0,006)$, la candidiasis pseudomembranosa $(p=0,009)$ y el estadio clínico $3(p=0,002)$. En lo que se refiere a tener candidiasis o estar colonizado, se determinó la asociación de los siguientes factores (Tabla 2$)$ : el género $(\mathrm{p}=0,011)$, la edad $(\mathrm{p}=0,005)$, carga viral $(\mathrm{p}=0,004)$, infección por el VIH $(p=0,001)$ recuento de LTCD4+ $(p=0,002)$, el estadio clínico $1(p=0,007)$, tratamiento ARV $(p=0,014)$, esquema de tratamiento con $2 \operatorname{INTR}+1 \operatorname{INNTR}(p=0,041)$ y 3 a 10 años con terapia ARV $(p=0,01)$.

Considerando los casos significativos de candidiasis, se identificaron los factores de exposición asociados a las

Tabla 2. Características demográficas, de laboratorio y terapia anti-retroviral de un grupo de niños y adolescentes mexicanos con infección por VIH y cultivo oral positivo de Candida spp. respecto de la manifestación clínica de candidiasis

\begin{tabular}{|c|c|c|c|c|c|c|c|c|c|}
\hline \multirow{3}{*}{ Género } & \multirow{3}{*}{$\begin{array}{l}\text { Masculino } \\
\text { Femenino }\end{array}$} & \multicolumn{2}{|c|}{$\begin{array}{c}\text { Todos } \\
\mathrm{n}=15(\%)\end{array}$} & \multicolumn{2}{|c|}{$\begin{array}{l}\text { Colonización } \\
n=8 \quad(53 \%)\end{array}$} & \multicolumn{2}{|c|}{$\begin{array}{c}\text { Candidiasis } \\
n=7 \text { (47\%) }\end{array}$} & \multirow{3}{*}{$\begin{array}{c}\mathbf{p} \\
0,007\end{array}$} & \multirow[t]{2}{*}{$\begin{array}{c}\text { OR (IC 95\%) } \\
p<0,05\end{array}$} \\
\hline & & 6 & (40) & 6 & (40) & 0 & & & \\
\hline & & 9 & (60) & 2 & (13) & 7 & (47) & & $17,27(1,81-+\operatorname{lnf})$ \\
\hline \multicolumn{2}{|l|}{ Promedio de edad; \pm (años) } & 8,8 & $\pm 6,2$ & 7,5 & $\pm 3,1$ & 10,28 & $\pm 8,5$ & 0,005 & $1,07 \quad(0,41-0,90)$ \\
\hline \multirow{2}{*}{ Grupo de edad } & Niños & 11 & (73) & 7 & (46) & 4 & (27) & & \\
\hline & Adolescentes & 4 & (27) & 1 & $(7)$ & 3 & (20) & ns & --- \\
\hline \multicolumn{2}{|c|}{ Carga viral; rango intercuartil (copias ARN-VIH-1/mL) } & \multicolumn{2}{|c|}{$334.978 ; 20-4.800$} & \multicolumn{2}{|c|}{$29 ; 20-39$} & \multicolumn{2}{|c|}{$717.777 ; 4.800-1.670 .000$} & 0,004 & --- \\
\hline \multirow[t]{2}{*}{ Infección por el VIH } & $<200$ copias $/ \mathrm{mL}$ & 9 & $(60)$ & 8 & (53) & 1 & (7) & 0,001 & \\
\hline & $>200$ copias $/ \mathrm{mL}$ & 6 & (40) & 0 & & 6 & (40) & 0,001 & $29,14(2,92-+\operatorname{lnf})$ \\
\hline \multicolumn{2}{|c|}{ Recuento de LTCD4+; rango intercuartil (céls/ $\mu$ l) } & \multicolumn{2}{|c|}{$1.219 ; 410-1.072$} & \multicolumn{2}{|c|}{$1752 ; 741-2.816$} & \multicolumn{2}{|c|}{$610 ; 83-1.065$} & 0,002 & \\
\hline \multirow[t]{3}{*}{ Estadio clínico } & Estadio clínico 1 & 6 & $(40)$ & 6 & $(40)$ & 0 & & 0,007 & \\
\hline & Estadio clínico 2 & 1 & (7) & 0 & & 1 & (7) & ns & $1,14 \quad(0,02-+\operatorname{lnf})$ \\
\hline & Estadio clínico 3 & 8 & (53) & 6 & (40) & 2 & (13) & & \\
\hline \multicolumn{2}{|l|}{ Tratamiento ARV (TARAA) } & 13 & (87) & 6 & $(40)$ & 7 & (47) & 0,014 & \\
\hline \multirow[t]{4}{*}{ Esquema TARAA } & Sin tratamiento & 2 & (13) & 2 & (13) & 0 & & ns & \\
\hline & 2 INTR + 1 IP & 4 & (27) & 4 & (27) & 0 & & 0,077 & \\
\hline & 3 INTR + 2 IP & 1 & (7) & 0 & & 1 & (7) & & $1,14(0,02-+\ln f)$ \\
\hline & 2 INTR + 1 INNTR & 8 & (53) & 2 & (13) & 6 & (40) & 0,041 & \\
\hline \multirow[t]{3}{*}{ Tiempo de terapia (años) } & $<3$ años & 3 & (20) & 0 & & 3 & (20) & 0,077 & $5,81 \quad(0,53-+\operatorname{lnf})$ \\
\hline & $3-10$ años & 8 & (53) & 7 & (47) & 1 & (6) & 0,010 & \\
\hline & $>10$ años & 4 & (27) & 1 & (6) & 3 & (20) & ns & \\
\hline
\end{tabular}




\begin{tabular}{|c|c|c|c|c|c|c|}
\hline \multirow[t]{2}{*}{ Factor } & \multicolumn{3}{|c|}{ Manifestaciones orales $(n=14)$} & \multicolumn{3}{|c|}{ Candidiasis pseudomembranosa $(n=3)$} \\
\hline & OR & (IC 95\%) & p & OR & (IC 95\%) & p \\
\hline Falla virológica CV > 200 copias/mL & 10,36 & $(0,99-550,97)$ & 0,050 & 85,12 & $(9,10-+\operatorname{lnf})$ & 0,000 \\
\hline Edad de esquema TARAA 3-10 años & 0,59 & $(0,10-3,08)$ & 0,715 & 0,08 & $(0,00-0,67)$ & 0,016 \\
\hline Esquema 2 INTR + 1 IP & 0,14 & $(0,01-0,94)$ & 0,042 & 0,16 & $(0,0-1,29)$ & 0,091 \\
\hline Esquema 2 INTR + 1 INNTR & 23,56 & $(2,35-1259,19)$ & 0,002 & 30,87 & $(3,73-+\operatorname{lnf})$ & 0,001 \\
\hline Tiempo de terapia (años) & 1,51 & $(1,09-2,22)$ & 0,009 & 1,18 & $(0,82-1,75)$ & 0,388 \\
\hline
\end{tabular}

$p=$ nivel de significancia estadística de 0,05 para prueba exacta de Fisher; $O R=$ razón de posibilidades; IC $=$ intervalo de confianza; $A R V=$ anti-retrovirales; INTR = inhibidor nucleósido de transcriptasa reversa; IP = inhibidor de proteasa; INNTR = inhibidor no nucleósido de la transcriptasa reversa; TARAA = tratamiento antiretroviral de alta actividad; + Inf $=$ límite superior desconocido (infinito positivo) para el IC.

manifestaciones orales y a la candidiasis pseudomembranosa (Tabla 3 ), estimándose que es 22 veces más probable tener una manifestación oral en esquemas de tratamiento con un INNTR $(\mathrm{p}=0,002)$ y 0,5 veces más probable debido al tiempo de la terapia $(\mathrm{p}=0,009)$. Además, se determinó que es 84 veces más probable tener candidiasis pseudomembranosa en falla virológica $(p=0,000)$ y 30 veces más probable cuando la terapia incluía un INNTR $(\mathrm{p}=0,001)$. Además, se estimó la influencia entre un factor de exposición y otro (Tabla 4), donde se observó que la posibilidad de tener manifestaciones orales se incrementó 88 veces $(\mathrm{p}=0,000)$ debido al tiempo con esquemas INTR+ 1 INNTR y 11 veces por cada año de terapia con ese mismo esquema $(p=0,000)$. La posibilidad de tener un caso de candidiasis oral se incrementó 0,6 veces por cada año de terapia $\operatorname{ARV}(p=0,020)$, y 1,6 veces en los pacientes con terapia ARV dado la duración del TARAA $(\mathrm{p}=0,038)$.

\section{Respuesta in vitro a antifúngicos de las especies aisladas de Candida spp}

Las especies de Candida mostraron diferencias en la respuesta a los antifúngicos, destacando $5 \mathrm{FC}$ con CIM de $4 \mu \mathrm{g} / \mathrm{mL}$ en $11(73 \%)$ cepas $(p=0,029)$ y FCA con CIM superior a $64 \mu \mathrm{g} / \mathrm{mL}$ en $10(66 \%)$ cepas $(\mathrm{p}=0,015)$. Para AMB se determinó un ECV igual o mayor a $2 \mu \mathrm{g} / \mathrm{mL}$ en todas las especies, asociado a resistencia de acuerdo a los puntos de corte establecidos por el CLSI (Tabla 5).

En cuanto a los factores asociados a la respuesta a los antifúngicos (Tabla 6), se observó que no hay diferencia en la CIM de 5FC, FCA e ITR ( $p>0,05)$ entre las especies colonizantes y las aisladas en casos de candidiasis. En el caso contrario, se observaron diferencias en AMB con CIM igual o superior a $2 \mu \mathrm{g} / \mathrm{mL}$ en todas las especies aisladas de candidiasis $(\mathrm{p}=0,031)$. Se encontró una CIM

\begin{tabular}{|c|c|c|c|}
\hline Manifestaciones orales $(n=14)$ & OR & (IC 95\%) & p \\
\hline Esquema ARV & 22,02 & $(2,83-+\operatorname{lnf})$ & 0,001 \\
\hline Tiempo de terapia (años) & 2,04 & $(1,17-4,93)$ & 0,005 \\
\hline Esquema INTR+ 1 INNTR & 88,94 & $(3,86-+\operatorname{lnf})$ & 0,000 \\
\hline Tiempo de terapia (años) & 11,76 & $(2,70-+\operatorname{lnf})$ & 0,000 \\
\hline \multicolumn{4}{|l|}{ Candidiasis oral $(n=7)$} \\
\hline Esquema ARV & 2,60 & $(1,12-10,96)$ & 0,020 \\
\hline Tiempo de terapia (años) & 1,59 & $(1,02-2,65)$ & 0,038 \\
\hline \multicolumn{4}{|c|}{$\begin{array}{l}\mathrm{p}=\text { nivel de significancia estadística de } 0,05 ; \mathrm{OR}=\text { razón de posibilidades; } \mathrm{IC}=\text { intervalo de } \\
\text { confianza; } \mathrm{ARV}=\text { anti-retrovirales; INTR= inhibidor nucleósido de transcriptasa reversa; INNTR = } \\
\text { inhibidor no nucleósido de la transcriptasa reversa; + Inf= límite superior desconocido (infinitc } \\
\text { positivo) para el IC. }\end{array}$} \\
\hline
\end{tabular}

de $\operatorname{VOR} \leq 0,125 \mu \mathrm{g} / \mathrm{mL}$ en las dos especies de niños sin TARAA y CIM superiores en especies de niños con TARAA $(p=0,029)$. Se observaron diferencias en la respuesta a VOR asociadas al tiempo de la terapia con CIM por arriba de $0,25 \mu \mathrm{g} / \mathrm{mL}$ después de dos años con tratamiento $\operatorname{ARV}(\mathrm{p}=0,012)$.

\section{Discusión}

La población pediátrica estudiada es una muestra homogénea (institucionalizada) con alta adherencia a los tratamientos, una dieta similar y con vigilancia clínica, lo que constituye una fortaleza del estudio. Además, nos proporciona información del comportamiento de infecciones oportunistas orales relacionadas a la infección por VIH en una comunidad extrahospitalaria ${ }^{21}$. 
Tabla 5. Distribución de la concentración inhibitoria mínima $(\mu \mathrm{g} / \mathrm{mL})$ en las especies de Candida aisladas de la mucosa oral

\begin{tabular}{|c|c|c|c|c|c|c|c|c|c|c|c|c|c|}
\hline \multirow[b]{2}{*}{ Antifúngico } & \multicolumn{12}{|c|}{ Número de aislamientos, CIM $(\mu \mathrm{g} / \mathrm{mL})$} & \multirow[t]{2}{*}{ p } \\
\hline & $n=15$ & $\leq 0,125$ & 0,25 & 0,5 & 1 & 2 & 4 & 8 & 16 & 32 & 64 & $\geq 128$ & \\
\hline Flucitosina & & & & & & & & & & & & & 0,029 \\
\hline C. albicans & 4 & & & & & & 3 & & & 1 & & & \\
\hline C. glabrata & 5 & & & & & & 5 & & & & & & \\
\hline C. kefyr & 2 & & & & & & 2 & & & & & & \\
\hline C. krusei & 2 & & & & & & & & & 2 & & & \\
\hline C. lipolytica & 1 & & & & & & & & & 1 & & & \\
\hline C. tropicalis & 1 & & & & & & 1 & & & & & & \\
\hline Anfotericina B & & & & & & & & & & & & & 0,380 \\
\hline C. albicans & 4 & & & & & 1 & & 1 & 2 & & & & \\
\hline C. glabrata & 5 & & & & & 3 & 1 & & 1 & & & & \\
\hline C. kefyr & 2 & & & & & & & & 2 & & & & \\
\hline C. krusei & 2 & & & & & 2 & & & & & & & \\
\hline C. lipolytica & 1 & & & & & & 1 & & & & & & \\
\hline C. tropicalis & 1 & & & & & 1 & & & & & & & \\
\hline Fluconazol & & & & & & & & & & & & & 0,015 \\
\hline C. albicans & 4 & & & & & & & & & & & 4 & \\
\hline C. glabrata & 5 & & & & & & 1 & 1 & & & 3 & & \\
\hline C. kefyr & 2 & & & & & & & 1 & & & 1 & & \\
\hline C. krusei & 2 & & & & & & & & & 1 & 1 & & \\
\hline C. lipolytica & 1 & & & & & & 1 & & & & & & \\
\hline C. tropicalis & 1 & & & & & & & & & & 1 & & \\
\hline Itraconazol & & & & & & & & & & & & & 0,322 \\
\hline C. albicans & 4 & & & & & 2 & 2 & & & & & & \\
\hline C. glabrata & 5 & & 1 & & 1 & 1 & 2 & & & & & & \\
\hline C. kefyr & 2 & & 2 & & & & & & & & & & \\
\hline C. krusei & 2 & & 2 & & & & & & & & & & \\
\hline C. lipolytica & 1 & & 1 & & & & & & & & & & \\
\hline C. tropicalis & 1 & & & & & & 1 & & & & & & \\
\hline Voriconazol & & & & & & & & & & & & & 0,055 \\
\hline C. albicans & 4 & & & 1 & & & 1 & 2 & & & & & \\
\hline C. glabrata & 5 & & & 3 & & & 2 & & & & & & \\
\hline C. kefyr & 2 & 1 & & & & & 1 & & & & & & \\
\hline C. krusei & 2 & & 2 & & & & & & & & & & \\
\hline C. lipolytica & 1 & 1 & & & & & & & & & & & \\
\hline C. tropicalis & 1 & & & & & & & 1 & & & & & \\
\hline
\end{tabular}

$\mathrm{CIM}=$ concentración inhibitoria mínima; $\mathrm{p}=$ nivel de significancia estadística de 0,05 para prueba exacta de Fisher.

Las especies prevalentes fueron C. glabrata $(33 \%)$, seguida por C. albicans (27\%). En este estudio se encontró una mayor frecuencia para C. glabrata; sin embargo, otros estudios demuestran que la especie prevalente en niños y adultos con infección por VIH es C. albicans, asociada principalmente a una disminución en el recuento de LTCD4+, al uso previo de antifúngicos y a cambios en el ambiente oral.

El análisis de asociación no mostró diferencias significativas entre las especies aisladas y los factores estudiados $(p>0,05)$, edad, género, estado inmunológico, manifestaciones orales, estadio clínico o terapia $\mathrm{ARV}^{22,23}$. Cabe señalar que todos los varones de esta población tuvieron especies colonizantes $(p=0,007)$, mientras que la probabilidad de tener un caso de candidiasis fue más alta en las mujeres (OR: 17,27; IC 95\% 1,81- +Inf). No obstante que la colonización es más frecuente en los hombres y la candidiasis en las mujeres, no se estableció 
Tabla 6. Factores asociados a la CIM de los antifúngicos en las especies de Candida spp. aisladas de la mucosa oral

\begin{tabular}{|c|c|c|c|c|c|c|c|c|c|c|c|c|}
\hline \multirow[b]{2}{*}{$\begin{array}{l}\text { Antifúngico } \\
\text { CIM }(\mu \mathrm{g} / \mathrm{mL})\end{array}$} & \multirow[b]{2}{*}{$\begin{array}{c}\text { Colonizantes } \\
\qquad n=8\end{array}$} & \multirow[b]{2}{*}{$\begin{array}{l}\text { Candidiasis } \\
\qquad n=7\end{array}$} & \multirow[b]{2}{*}{$p$} & \multicolumn{2}{|c|}{ Esquema } & \multicolumn{7}{|c|}{ Tiempo con esquema ARV } \\
\hline & & & & $\begin{array}{l}\text { TARAA } \\
n=13\end{array}$ & $\begin{array}{l}\text { Naïve } \\
n=2\end{array}$ & p & $\begin{array}{c}0 \\
n=2\end{array}$ & $\begin{array}{c}2 \\
n=4\end{array}$ & $\begin{array}{c}3 \\
n=2\end{array}$ & $\begin{array}{c}5 \\
n=1\end{array}$ & $\begin{array}{c}7 \\
n=6\end{array}$ & p \\
\hline \multicolumn{13}{|l|}{$5 F C$} \\
\hline 4 & 6 & 5 & 1,00 & 10 & 1 & 0,476 & 1 & 3 & 2 & 1 & 4 & 1,00 \\
\hline 32 & 2 & 2 & & 1 & 1 & & 1 & 1 & 0 & 0 & 2 & \\
\hline \multicolumn{13}{|l|}{ AMB } \\
\hline 2 & 1 & 6 & 0,031 & 7 & 0 & 0,333 & 0 & 2 & 0 & 1 & 4 & 0,40 \\
\hline 4 & 2 & 0 & & 1 & 1 & & 1 & 1 & 0 & 0 & 0 & \\
\hline 8 & 1 & 0 & & 1 & 0 & & 0 & 0 & 1 & 0 & 0 & \\
\hline 16 & 4 & 1 & & 14 & 1 & & 1 & 1 & 1 & 0 & 2 & \\
\hline \multicolumn{13}{|l|}{ FCA } \\
\hline 4 & 1 & 1 & 1,00 & 1 & 1 & 0,133 & 1 & 0 & 0 & 1 & 0 & 0,576 \\
\hline 8 & 1 & 1 & & 1 & 1 & & 1 & 1 & 0 & 0 & 0 & \\
\hline 32 & 1 & 0 & & 1 & 0 & & 0 & 0 & 0 & 0 & 1 & \\
\hline 64 & 3 & 3 & & 6 & 0 & & 0 & 2 & 1 & 0 & 3 & \\
\hline$\geq 128$ & 2 & 2 & & 4 & 0 & & 0 & 1 & 1 & 0 & 2 & \\
\hline \multicolumn{13}{|l|}{ ITR } \\
\hline 0,25 & 4 & 2 & 0,720 & 4 & 2 & 0,543 & 2 & 0 & 0 & 1 & 3 & 0,153 \\
\hline 1 & 0 & 1 & & 1 & 0 & & 0 & 1 & 0 & 0 & 0 & \\
\hline 2 & 2 & 1 & & 3 & 0 & & 0 & 2 & 1 & 0 & 0 & \\
\hline 4 & 2 & 3 & & 5 & 0 & & 0 & 1 & 1 & 0 & 3 & \\
\hline \multicolumn{13}{|l|}{ VOR } \\
\hline$\leq 0,125$ & 2 & 0 & 0,217 & 0 & 2 & 0,029 & 2 & 0 & 0 & 0 & 0 & 0,012 \\
\hline 0,25 & 1 & 1 & & 2 & 0 & & 0 & 0 & 0 & 0 & 2 & \\
\hline 0,5 & 1 & 3 & & 4 & 0 & & 0 & 3 & 0 & 1 & 0 & \\
\hline 4 & 4 & 1 & & 5 & 0 & & 0 & 1 & 2 & 0 & 2 & \\
\hline 8 & 0 & 2 & & 2 & 0 & & 0 & 0 & 0 & 0 & 2 & \\
\hline
\end{tabular}

$5 F C=$ fluocitosina; $A M B=$ anfotericina $B ; F C A=$ fluconazol; $I T R=$ itraconazol; $V O R=$ voriconazol; $p=$ nivel de significancia estadística de 0,05 para prueba exacta de Fisher; $A R V=$ anti-retroviral, $T A R A A=$ tratamiento anti-retroviral de alta actividad.

una relación del género con los recuentos de LTCD4+ asociados a inmunosupresión $(\mathrm{p}>0,4)$, lo que podría sugerir que las diferencias de género en los niños y adolescentes podría ser una variable por estudiar debido a su influencia en los mecanismos de inmunidad del hospedero o de patogenicidad de las especies de Candida $a^{4,23,24}$.

Todos los casos del estadio 3 resultaron positivos al cultivo de Candida (OR: 19,72; IC 95\% 2,58- +Inf). El $40 \%$ de las especies colonizantes se aisló de casos en estadio clínico $3(\mathrm{p}=0,041)$, opuesto a lo que se esperaba, ya que la manifestación por candidiasis oral es un criterio para la etapificación clínica en la categoría $3^{19}$ y por tal motivo podríamos esperar más casos de candidiasis que de colonización.

Las infecciones orales oportunistas son útiles como indicador de la progresión de la infección por VIH en los servicios de salud que carecen de pruebas para el seguimiento del estado inmunológico de los pacientes ${ }^{6}$. En este estudio se observó que las manifestaciones orales fueron más frecuentes en casos con falla virológica $(\mathrm{p}=0,031)$, en particular la candidiasis pseudomembranosa $(p=0,009)$. No se encontró asociación estadísticamente significativa entre la caries, la estomatitis aftosa o el absceso recurrente y el aislamiento de Candida sp. $(p>0,05)$. De manera similar a otros estudios que miden el efecto del TARAA, se determinó un aumento de los casos de las manifestaciones orales cuando la concentración de LTCD4+ disminuye y la CV aumenta por arriba de 200 copias $/ \mathrm{mL}^{4,6}$. No se observó diferencia en la colonización, manifestaciones orales o especies de Candida entre los sujetos con TARAA o sin ella; sin embargo, $57 \%$ de los casos con colonización tenían tres 
años o menos con terapia ARV y se encontraban bajo un esquema TARAA de primera línea.

El tiempo de terapia es un factor que influye en las manifestaciones orales de sujetos con TARAA, en específico cuando el esquema incluye a un INNTR ${ }^{22}$. En los resultados de este estudio se observó una alta posibilidad de tener manifestaciones orales en esquemas de primera línea con INNTR (OR: 23,56; IC 95\% 2,35-1.259,19), aunque en tratamientos de primera línea con IP se observó un efecto protector (OR: 0,14; IC 95\% 0,01- 0,94) . $^{4}$ Las manifestaciones orales se podrían utilizar como un indicador de falla terapéutica de los fármacos de primera línea con INNTR.

En este estudio se determinó una CIM $\geq 128 \mu \mathrm{g} / \mathrm{mL}$ para FCA en los aislados de C. albicans (80\%) superior al ECV que sugiere resistencia $(p=0,001)$, a diferencia de otros estudios en poblaciones mexicanas, donde ClarkOrdóñez y cols ${ }^{25}$ encontraron especies resistentes a FCA entre 0,8 y 3,1\% y Sánchez-Vargas y cols ${ }^{26}$ en $2,2 \%$ de los aislados de C. albicans. Se determinó que las especies no-albicans tienen CIM de FCA $\leq 64 \mu \mathrm{g} / \mathrm{mL}$. Por otra parte, Sánchez-Vargas y cols ${ }^{26}$ no reporta resistencia a AMB en especies colonizantes y Clark-Ordóñez ${ }^{25}$ sólo en $6 \%$ de los aislados. Este estudio evidenció que la totalidad de especies aisladas de la mucosa oral tenían un ECV para $\mathrm{AMB} \geq 2 \mu \mathrm{g} / \mathrm{mL}$, que sugiere la presencia de mecanismos de resistencia al antifúngico en todas las cepas estudiadas. Es importante destacar el aislamiento de C. lipolytica como especie colonizante en un paciente sin tratamiento ARV (naïve), con una $\mathrm{CV}<50$ copias/mL y concentración de LTCD4+> 500 céls $/ \mu \mathrm{L}$, con CIM de FCA $\leq 4 \mu \mathrm{g} / \mathrm{mL}$, ITR $\leq 0,25 \mu \mathrm{g} / \mathrm{mL}$ y VOR $\leq 0,125 \mu \mathrm{g} / \mathrm{mL}$; considerado un patógeno emergente ${ }^{27}$. Los antecedentes en el uso de antifúngicos nos ayudarían a entender los factores de exposición que intervienen en los mecanismos de resistencia de las especies de Candida $^{14,28}$; no obstante, estos niños carecen de historia clínica previa a su institucionalización y por lo tanto no se conoce esta información.

Se identificaron especies con ECV que sugieren resistencia a ITR (46\%) en aislados provenientes de pacientes bajo esquema con INNTR ( $p=0,041)$. Jaruratanasirikul y Sriwiriyajan en el año 2007, demostraron que nevirapina funciona como inductor de la enzima citocromo P450, que acelera el metabolismo del ITR y disminuye su concentración plasmática, por lo cual recomiendan realizar ajuste de dosis cuando se administran al mismo tiempo ${ }^{29}$. Es importante considerar las interacciones farmacológicas, las reacciones adversas (xerostomía) y la farmacocinética de los medicamentos que se administren en conjunto con los ARV, debido a que el efecto se puede potenciar o reducir.

Agradecimientos. Esta investigación fue apoyada por la 17 Convocatoria Interna de Proyectos de Investigación de la Universidad Autónoma de Baja California. Queremos agradecer a la Srta. Juana Ortiz Quezada, directora y fundadora de la Casa Hogar Eunime por Tijuana por todas las facilidades otorgadas durante el estudio, así como al Hospital General de Tijuana y al Dr. Graciano López Espinoza.

\section{Resumen}

Introducción: Se desconocen los factores asociados a la candidiasis oral en población pediátrica con infección por VIH de los países en desarrollo. Objetivo: Identificar los factores asociados a la colonización por Candida, candidiasis oral y la susceptibilidad in vitro a antifúngicos, en niños y adolescentes con infección por VIH institucionalizados en la ciudad de Tijuana, México. Materiales y Métodos: Se examinó la cavidad oral de 30 niños y adolescentes con infección por VIH, se obtuvo una muestra de la mucosa oral para identificar las especies de Candida mediante cultivo y auxonograma. La susceptibilidad a los antifúngicos se determinó de acuerdo al CLSI. Los indicadores del estado inmunológico y falla virológica se clasificaron conforme a la OMS. Resultados: Se identificaron seis especies de Candida, $53 \%$ colonizantes y $47 \%$ causantes de candidiasis. Los factores asociados a candidiasis fueron alta carga viral $(\mathrm{p}=0,001)$, menor recuento de LTCD4+ $(\mathrm{p}=0,002)$ y esquema TARAA $(\mathrm{p} \leq 0,014)$. La especie prevalente fue C. glabrata (33\%); sin embargo, C. albicans $(27 \%)$ fue más resistente a fluconazol $(\mathrm{p}=0,001)$. Las especies resistentes a itraconazol se identificaron en esquemas que incluyen un INNTR $(p=0,041)$. Conclusiones: Los niños y adolescentes con infección por VIH institucionalizados mostraron una prevalencia elevada de Candida spp. colonizante y resistencia a los antifúngicos relacionada con los INNTR.

\section{Referencias bibliográficas}

1.- UNAIDS, Joint United Nations Programm on HIV/AIDS. AIDS by the numbers [Internet]. UNAIDS, Geneva Switzerland; 2016 [citado el 18 de enero de 2017]. Disponible en: http:// www.unaids.org/sites/default/files/media_asset/ AIDS-by-the-numbers-2016_en.pdf
2.- Vigilancia Epidemiológica de casos de VIH/ SIDA en México, Registro Nacional de Casos de VIH y sida [base de datos]. 3er trimestre de 2016. Secretaria de Salud, Dirección General de Epidemiología, CENSIDA; 2016 [citado el 1 de mayo de 2017]. Disponible en: http://www. censida.salud.gob.mx/descargas/epidemiologia/ RN_3er_trim_2016_1.pdf
3.- Notificación Semanal Casos Nuevos de Enfermedades 2017 [base de datos] Reporte correspondiente a febrero de 2017. Secretaria de Salud, Dirección General de Epidemiología, Estados Unidos Mexicanos; 2017 [citado el 1 de mayo de 2017]. Disponible en: https://www. gob.mx/cms/uploads/attachment/file/201651/2 Reporte_de_febrero_2017.pdf 
4.- Cerqueira D F, Portela M B, Pomarico L, de Araújo Soares R M, de Souza I P R, Castro G F. Oral Candida colonization and its relation with predisposing factors in HIV-infected children and their uninfected siblings in Brazil: the era of highly active antiretroviral therapy. J Oral Pathol Med. 2010; 39 (2): 188-94. doi: 10.1111/j.1600-0714.2009.00857.x. Epub 2009 Dec 22.

5.- Gaitán-Cepeda L A, Martínez-González M, Ceballos-Salobreña A. Oral candidosis as a clinical marker of immune failure in patients with HIV/AIDS on HAART. AIDS Patient Care STDs. 2005; 19 (2): 70- DOI:10.1089/ apc.2005.19.70

6.- Yengopal V, Bhayat A, Coogan M. Pediatric oral HIV research in the developing world. Adv Dent Res. 2011; 23(1): 61-6. doi: 10.1177/0022034511399287.

7.- Alarcón J O, Freimanis-Hance L, Krauss M, Reyes M F, Cardoso C A A, Mussi-Pinhata M $\mathrm{M}$, et al. Opportunistic and other infections in HIV-Infected children in Latin America compared to a similar cohort in the United States. AIDS Res Hum Retroviruses. 2012; 28 (3): 282-8. doi: 10.1089/AID.2011.0057. Epub 2011 Sep 19.

8.- Diz Dios P, Scully C. Antiretroviral therapy: effects on orofacial health and health care. Oral Dis. 2014; 20 (2): 136-45. doi: 10.1111/ odi.12093. Epub 2013 Mar 27.

9.- L'homme R, Warris A, Gibb D, Burger D. Children with HIV are not small adults: what is different in pharmacology? Curr Opin HIV AIDS. 2007; 2 (5): 405-9. doi: 10.1097/ COH.0b013e3282ced13f.

10.- Yengopal V, Kolisa Y, Thekiso M, Molete M. The child and adolescent with HIV in resource poor countries. Oral Dis. 2016; 22: 25-34. doi: 10.1111/odi.12411. Epub 2016 Feb 16.

11.- Alastruey-Izquierdo A, Melhem M S C, Bonfietti L X, Rodríguez-Tudela J L. Susceptibility test for fungi: clinical and laboratorial correlations in medical mycology. Rev Inst Med Trop São Paulo. 2015; 57 (Suppl 19): 57-64. doi: 10.1590/S003646652015000700011.

12.- White T C, Holleman S, Dy F, Mirels L F, Stevens D A. Resistance Mechanisms in clinical isolates of Candida albicans. Antimicrob Agents Chemother. 2002; 46 (6): 1704-13.

13.- Migliorati C A, Birman E G, Cury A E.
Oropharyngeal candidiasis in HIV-infected patients under treatment with protease inhibitors. Oral Surg Oral Med Oral Pathol Oral Radiol Endod. 2004; 98 (3): 301-10.

14.- Delgado A C D, De Jesus Pedro R, Aoki F H, Resende M R, Trabasso P, Colombo A L, et al. Clinical and microbiological assessment of patients with a long-term diagnosis of human immunodeficiency virus infection and Candida oral colonization. Clin Microbiol Infect. 2009; 15 (4): 364-71.

15.- Clinical and Laboratory Standards Institute. Reference Method for Broth Dilution Antifungal Susceptibility Testing of Yeasts: Approved Standard. 3rd ed. Wayne, PA: National Committee for Clinical Laboratory Standards; 2008

16.- Clinical and Laboratory Standards Institute. Performance Standards for Antifungal Susceptibility Testing of Yeasts. 1st ed. CLSI supplement M60. Wayne, PA: National Committee for Clinical Laboratory Standards; 2017.

17.- Clinical and Laboratory Standards Institute. Epidemiological cutoff values for antifungal susceptibility testing. 1st ed. CLSI supplement M59. Wayne, PA: National Committee for Clinical Laboratory Standards; 2016.

18.- Inter-Agency Task Team (IATT) for Prevention and Treatment of HIV Infection in Pregnant Women, Mother and Children, World Health Organization, UNICEF. IATT paediatric ARV formulary and limited-use list: 2016 update [Internet]. World Health Organization, UNICEF; 2016 [citado el 20 de marzo de 2017]. Disponible en: http://www.who.int/hiv/ pub/paediatric/iatt-paediatric-hiv-2016/en/

19.- Centers for Disease Control and Prevention (CDC). Revised surveillance case definition for HIV infection-United States, 2014. Morbid Mortal Wkly Rep MMWR Recomm Rep. 2014; 63 (RR-03): 1-10.

20.- Panel on Antiretroviral Therapy and Medical Management of Children Living with HIV. Guidelines for the Use of Antiretroviral Agents in Pediatric HIV Infection [Internet]. Guidelines for the Use of Antiretroviral Agents in Pediatric HIV Infection. 2017 [citado el 20 de marzo de 2017]. Disponible en: http:// aidsinfo.nih.gov/contentfiles/lvguidelines/ pediatricguidelines.pdf

21.- Blignaut E. Oral candidiasis and oral yeast carriage among institutionalised South African paediatric HIV/AIDS patients. Mycopathologia. 2007; 163 (2): 67-73. DOI:10.1007/s11046006-0087-9

22.- Pomarico L, de Araújo Castro G F B, de Souza I P R, Oliscovicz N F. Effect of highly active antiretroviral therapy use on oral manifestations in pediatric patients infected with HIV. Indian J Dent Res. 2015; 26 (2): 200-4.

23.- Pomarico L, Cerqueira D F, de Araujo Soares R M, de Souza I P R, de Araujo Castro G F $\mathrm{B}$, Socransky S, et al. Associations among the use of highly active antiretroviral therapy, oral candidiasis, oral Candida species and salivary immunoglobulin A in HIV-infected children. Oral Surg Oral Med Oral Pathol Oral Radiol Endod. 2009; 108 (2): 203-10.

24.- Klein S L, Flanagan K L. Sex differences in immune responses. Nat Rev Immunol. 2016; 16 (10): 626-38. doi: 10.1038/nri.2016.90. Epub 2016 Aug 22

25.- Clark-Ordóñez I, Callejas-Negrete O A, Aréchiga-Carvajal E T, Mouriño-Pérez R R. Candida species diversity and antifungal susceptibility patterns in oral samples of HIV/ AIDS patients in Baja California, Mexico. Med Mycol. 2017; 55 (3): 285-94. doi: 10.1093/ mmy/myw069.

26.- Sánchez-Vargas L O, Ortiz-López N G, Villar M, Moragues M D, Aguirre J M, Cashat-Cruz M, et al. Point prevalence, microbiology and antifungal susceptibility patterns of oral Candida isolates colonizing or infecting Mexican HIV/AIDS patients and healthy persons. Rev Iberoam Micol. 2005; 22 (2): 83-92.

27.- Trabelsi H, Chtara K, Khemakhem N, Néji S, Cheikhrouhou F, Sellami H, et al. Fungemia caused by Yarrowia lipolytica. Mycopathologia. 2015; 179 (5-6): 437-45. doi: 10.1007/s11046-015-9859-4. Epub 2015 Jan 23.

28.- de Paula S B, Morey A T, Santos J P, dos Santos P M C, Gameiro DG, Kerbauy G, et al. Oral Candida colonization in HIV-infected patients in Londrina-PR, Brazil: antifungal susceptibility and virulence factors. J Infect Dev Ctries. 2015; 9(12): 1350-9. doi: 10.3855/ jidc. 6970.

29.- Jaruratanasirikul S, Sriwiriyajan S. Pharmacokinetic study of the interaction between itraconazole and nevirapine. Eur J Clin Pharmacol. 2007; 63 (5): 451-6. DOI:10.1007/ s00228-007-0280-x. 